\title{
Association between intention to call EMS in stroke patients and level of hospital classification and emergency medical service usage among stroke patients in China
}

\author{
Yi-Xiu Lu ${ }^{1}$, Sheng-De Li ${ }^{1}$, Guang-Liang Shan ${ }^{2}$, Bin Peng ${ }^{1}$ \\ ${ }^{1}$ Department of Neurology, Peking Union Medical College Hospital, Peking Union Medical College and Chinese Academy \\ of Medical Sciences, Beijing, China \\ ${ }^{2}$ Institute of Basic Medical Sciences, Chinese Academy of Medical Sciences, Beijing, China
}

\begin{abstract}
Introduction. Different categories of hospitals in China have varying levels of patient education. Stroke recognition and emergency medical services (EMS) usage in patients appears to be closely associated with patient education in hospital. This study aimed to explore the effect of hospital classification in China on a patient's ability to recognise stroke symptoms and the likelihood of using the EMS.

Material and methods. A cross-sectional, community-based study was conducted from January to May 2017, and 1,426 residents who had previously been hospitalised were analysed. The patients involved in the study were from 69 administrative areas in China. Multivariable logistic regression models were developed separately for primary, secondary, and tertiary hospitals to identify the associations between hospital grades and patient stroke recognition or the prospects of them using the EMS.

Results. Among the 1,426 patients studied, 725 had been admitted to tertiary hospitals, 448 to secondary hospitals, and 253 to primary hospitals. According to univariate analysis, tertiary hospital patients were more likely to use the EMS than patients in primary and secondary hospitals. The difference therein was still significant after full adjustment.

Conclusions. Patients in tertiary hospitals were significantly more likely to use the EMS promptly compared to patients in primary or secondary hospitals. Therefore, patient education on timely EMS usage at stroke onset should be enhanced in primary and secondary hospitals in China.
\end{abstract}

Key words: secondary prevention, Chinese hospital grading system, patient education, awareness, emergency

(Neurol Neurochir Pol 2021; 55 (6): 582-591)

\section{Introduction}

Ischaemic stroke has been the leading cause of death and disability in China since 2008 [1-3]. Prompt and effective medical treatment has a beneficial impact on the prognosis and survival of stroke patients. Many guidelines and literature reports on stroke care recommend that the correct response to stroke onset is to use the emergency medical services (EMS). The ambulances play an important role in the efficient transfer of patients and act as mobile stroke units, which improves the efficiency of hospital emergency treatment of stroke patients [4]. Hence, patient awareness of stroke symptoms and the EMS is vital to ensure timely medical assistance and a favourable prognosis [5]. However, Chinese people still have poor awareness of stroke symptoms and treatment compared to people in developed countries [6]. It is very important to improve stroke knowledge among Chinese people.

In recent decades, there have been thousands of new hospitals built in China [7]. Chinese hospitals are classified into three grades: primary, secondary, and tertiary, according

Address for correspondence: Bin Peng, Department of Neurology, Peking Union Medical College Hospital, Peking Union Medical College and Chinese Academy of Medical Sciences, No. 9, Dongdan santiao, Dongcheng District, Beijing 100730, China; e-mail: pengbin3@hotmail.com 
to their ability to provide medical care and patient education, and perform medical research. A tertiary hospital is defined as a regional medical institution that provides comprehensive and specialist health services [8].

However, very little research has focused on the effect of hospital classification on patient awareness of stroke symptoms. The ability of patients to recognise and respond correctly to stroke onset is an effective indicator for the indirect assessment of stroke education. Hence, stroke patients with previous hospitalisation experience should make for a suitable sample group because of the possibility that they have received stroke education in the hospital. Therefore, this study was aimed at evaluating the health education status of stroke patients admitted to different classifications of hospitals in China, by measuring their ability to recognise stroke symptoms and their intent to use the EMS.

\section{Material and methods}

\section{Study design and participants}

This study was based on the FAST-RIGHT study, which is a part of the China National Stroke Screening Survey (CNSSS) [9], the details of which are thoroughly set out on the CNSSS official website [10]. The CNSSS was a nationwide, cross-sectional, community-based survey with a two-stage, stratified sampling framework based on county-level demographic data.

Residents underwent screening assessments for our study between 1 January, 2017 and 31 May, 2017 [11].

The criterion for participants was as follows: residents aged 40 years or older in each community of the 221 administrative areas located in 31 provinces of mainland China [11]. Only residents aged 40 years and older were screened, and those who had a history of cerebral vascular disease (CVD) between 2014 and 2017 were enlisted [11].

The FAST-RIGHT study gathered a subgroup of residents from 69 administrative areas; most provinces were accounted for in the subgroup. Face-to-face interviews were conducted to ensure credibility, accuracy, and integrity. In addition to the standard questionnaire, four supplementary questions were asked relating to the interviewee's awareness of the main stroke symptoms and actions connected to them (see the supplementary material for details).

The FAST-RIGHT study was approved by the central ethics committee of the Peking Union Medical College Hospital, the principal study centre. All participants provided written consent allowing access to their patient information.

\section{Procedure}

Trained staff conducted the assessments using a standard questionnaire, covering socio-demographics, family history, medical status, and lifestyle factors. In accordance with the FAST-RIGHT study, four questions regarding stroke awareness and associated responses were asked. The screening data from the questionnaires was transferred to an electronic database by an experienced data manager who examined it in detail for errors and completeness. After completing the questionnaire survey, the participants received stroke-related education.

A participant's unprompted recognition of facial drooping, arm weakness, and speech disturbance (slurred speech or word-finding difficulties) was regarded as an awareness of stroke symptoms [12]. Calling the EMS immediately after the onset of any of these symptoms was defined as the correct response to stroke symptoms. Risk factors evaluated in the questionnaire included hypertension, diabetes mellitus, dyslipidemia, overweight/obesity, history of atrial fibrillation or valvular heart disease, smoking, physical inactivity, and a family history of stroke, based on the standard definitions (see the online-only data supplement).

A total of 1,520 participants surveyed had experienced a recent (i.e. between 2014 and 2017) stroke. One was excluded for lack of official hospitalisation records; the other 1,519 had proven hospitalisation records between 2014 and 2017. Of these, 93 were hospitalised more than once during this period, while the remaining 1,426 had only one hospitalisation experience. There was no significant difference in the baseline data between those hospitalised once and those hospitalised more than once (see suppl. Tab. S1). The 1,426 participants who had experienced a stroke with a single episode of hospitalisation between 2014 and 2017 were chosen as the sample group for this study.

Demographic characteristics were classified by sex (male or female); age group (40-49, 50-59, 60-69, 70-79, or 80-99 years); education level (below middle school, middle school, high school, or college graduate); and personal income ( $\leq 5,000,5,000-20,000$, and $\geq 20,000$ yuan/month). The thirty-one provinces in mainland China were separated into two economic development groups according to their 2016 gross domestic product (GDP) per capita ranking (ranked as 1-11 and 12-31, as per the 2016 Provincial Statistical Bureau) [13]. In addition, hospitals were designated as primary, secondary, or tertiary institutions based on comprehensive hospital grading management standards [14] (see the online data supplement for further details on the Chinese hospital classification). This study considered four ways in which patients obtain medical care: hospitalisation, the involvement of emergency services, outpatient referral, and general practitioner (GP, family doctor). A modified Rankin scale (mRS) score was employed to measure the severity of the stroke among patients with different approaches.

\section{Statistical analysis}

Differences in the baseline characteristics of patients admitted to tertiary hospitals and those admitted to primary and secondary hospitals were assessed using $\chi 2$ tests. The stroke recognition rate (SRR) and the correct action rate (CAR) were assessed separately for each hospital category. Multivariable logistic regression was conducted to analyse the relationship between hospital classification, stroke awareness, and 


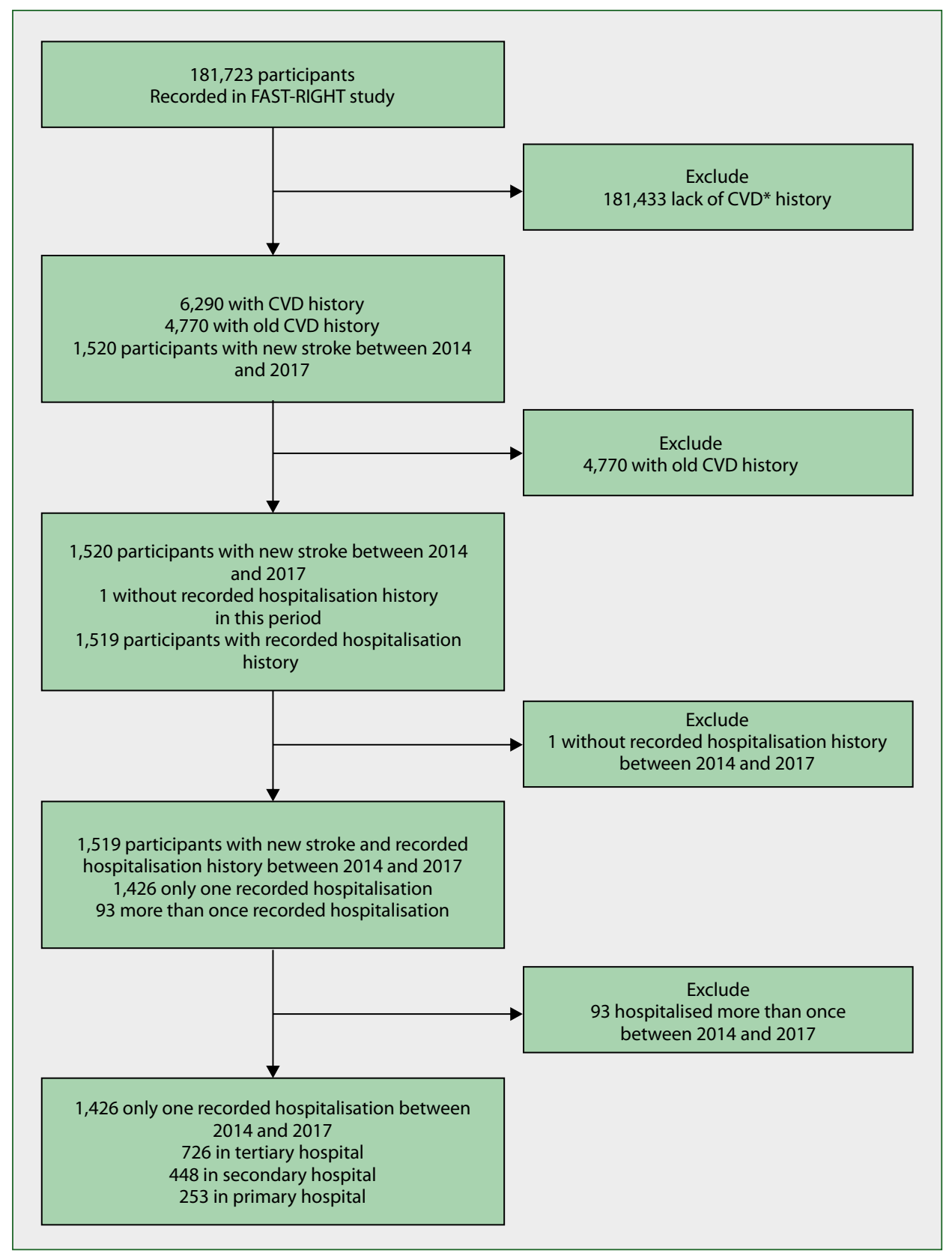

Figure 1. Flow chart of study selection. CVD - cerebrovascular disease

subsequent EMS usage. Additionally, different models were established to adjust for other potential confounding factors. All analyses were conducted using SAS version 9.3 software, taking a standard two-sided $\mathrm{p}$ value $(<0.05)$ as statistically significant.

\section{Results}

Of the 1,426 patients analysed in this study (Fig. 1), 725 were admitted to tertiary hospitals, 448 to secondary hospitals, and 253 to primary hospitals. Patient education level, monthly income, knowledge about stroke, the prevalence of stroke in their family or community, the ways in which they obtained medical care, mRS score, and the economic development of the province where the hospital was situated, all differed significantly between the patients in the different hospital classifications. The baseline data is set out in Table 1 . Patients in tertiary hospitals tended to have higher income and education levels than did those in primary and secondary hospitals. Moreover, the tertiary hospitals in this study were themselves inclined to be situated in provinces with higher GDP per capita. Patients in tertiary hospitals tended to have a higher mRS score compared to other patients, yet there was no significant difference in value. Furthermore, patients who 
used the EMS to seek medical care also did not have higher mRS scores than others (see suppl. Tab. S2).

Univariate analysis indicated that patients admitted to tertiary hospitals had a significantly higher CAR than patients admitted to primary and secondary hospitals, as set out in Table $2(79.6 \%$ vs. $53.0 \% / 60.7 \%, \mathrm{p}<0.0001)$. The tertiary hospital group was more inclined to use the EMS immediately after stroke onset, as evidenced by multivariate analysis. Moreover, this association was statistically significant in the final model, after full adjustment, as seen in Figure 2 (tertiary $v$ s. secondary OR: $0.44,95 \%$ CI $0.33-0.52, \mathrm{p}<0.0001$, Fig. 2; tertiary vs. primary OR: $0.39,95 \%$ CI $0.26-0.53$, $\mathrm{p}<0.0001$, Fig. 2).

Despite these results, and when compared to other hospitals, hospitalisation in tertiary hospitals did not increase the probability of recognising stroke symptoms consistently across the four different logistic models (Fig. 2). However, we observed that patients in emergency departments were significantly more likely (88.2\%) to use the EMS immediately than were patients under non-emergency care: in hospitalisation (67.0\%), as outpatients (69.9\%), or those who had seen a GP (42.9\%), as set out in Table 3.

In subgroup analysis of the tertiary hospital group, patients with a higher personal income also showed significantly higher stroke recognition than did those with lower incomes (91.2\% vs. $85.5 \%$ ), as illustrated in Table 4. Patients in provinces with higher GDP per capita were less likely to use the EMS at stroke onset than were those in lower GDP areas (74.4\% vs. 82.1\%), as shown in Table 4. In the baseline data of tertiary hospital patients who sought different means of obtaining medical care, emergency patients tended to have better knowledge about strokes than did others. Moreover, patients in economically developed provinces preferred to be outpatients rather than to use the EMS or other medical approaches (see suppl. Tab. S3). However, tertiary hospital patients with a higher educational level (college or above) did not have a significantly higher SRR or CAR than did patients with a lower educational level (high school or below), as shown in Table 4 .

\section{Discussion}

Our study reveals that hospital classification is significantly related to a patient's intent to use the EMS. Tertiary hospital patients were more likely to use the EMS following stroke onset than were primary or secondary hospital patients. This study is the first large-scale study in China to address this topic using a very representative sample. It is clear that tertiary hospitals are better equipped with more modern medical resources, and that the cost of staying in them is higher than in other hospitals $[15,16]$. Our findings indicate that primary or secondary hospital patients may not be as health aware as tertiary hospital patients, and may exhibit a lack of knowledge regarding stroke and EMS utilisation because of inadequate in-hospital education [17-19]. Prompt recognition of the symptoms of a stroke is essential. However, data from three cities in China reveals that public awareness of stroke symptoms and treatment remains poor, and that only $18.8 \%$ of people use the EMS at stroke onset [20]. In China, there are many shortcomings regarding public awareness of stroke symptoms and the proper course of action after stroke onset, although stroke-related in-hospital education has played a significant role in addressing these inadequacies [21]. Education conducted in hospitals has been shown to comprise $13-51 \%$ of the knowledge source for strokes $[22,23]$. The quality of facilities and in-hospital education in tertiary hospitals is much better than that in primary or secondary hospitals. As a result, patients in primary or secondary hospitals require better stroke-related education so as to improve their awareness of the correct response at stroke onset.

This study also revealed that there was no significant difference in the level of stroke awareness among patients in hospitals with different classification levels, which indicates that in-hospital education about stroke recognition must be improved in all hospital categories. Although many hospitals in China provide varied educational support (including paper-based materials, audio-visual materials, and lectures), patient knowledge about stroke is still lacking. Previous studies have confirmed that, at the time of discharge, most patients in China had little knowledge regarding strokes [24, 25]. Research has also shown that there is no significant correlation between patients' awareness of stroke signs and the likelihood of them using the EMS immediately at stroke onset $[25,26]$.

The data analysis in this study showed a significant difference in the CAR after stroke depending on which medical service the patient sought. Emergency patients were significantly more likely to use the EMS after stroke onset than were patients who used other facilities. The reasons for this are unclear. Because emergency medicine is a new specialism in China [14], many Chinese people may not know much about the convenience and suitability of EMS usage. However, patients admitted to hospitals in China via the EMS have a significantly higher rate of rt-PA usage rate than do patients admitted through other approaches (12.1\% vs. 2.3\%) [27]. In addition, emergency patients may know more about the benefit of EMS utilisation due to their previous EMS usage experience [4]. However, studies have shown that the rate of EMS usage after stroke onset in China is much lower than that in Western countries [25, 28, 29]. This finding suggests that China needs more health education campaigns to increase public awareness about the benefits of obtaining immediate EMS support.

In this study, SRR ( $85.7 \%$ vs. $81.7 \%)$ and CAR $(68.9 \%$ vs. $60.9 \%$ ) in patients with a history of CVD seemed to be higher than that of other participants in the FAST-RIGHT study [11]. Thus, the increased awareness of CVD patients regarding stroke symptoms and the correct response might be related to their experience of stroke. However, the difference between patients with and without a history of CVD in SRR 
Table 1. Baseline data of patients in different grades of hospital in China

\begin{tabular}{|c|c|c|c|c|}
\hline Patients & $\begin{array}{l}\text { Tertiary } \\
\text { n/N (\%) }\end{array}$ & $\begin{array}{l}\text { Secondary } \\
\text { n/N (\%) }\end{array}$ & $\begin{array}{c}\text { Primary hospitals } \\
n / N(\%)\end{array}$ & P-value \\
\hline Age [years] & & & & 0.2259 \\
\hline $40-49$ & $33 / 725(4.6)$ & $12 / 448(2.7)$ & $11 / 253(4.3)$ & \\
\hline $50-59$ & $124 / 725(17.1)$ & 66/448 (14.7) & $46 / 253(18.2)$ & \\
\hline $60-69$ & 263/725 (36.3) & $173 / 448$ (38.6) & $84 / 253(33.2)$ & \\
\hline $70-79$ & $225 / 725(31.0)$ & $133 / 448(29.7)$ & $88 / 253(34.8)$ & \\
\hline $80-99$ & $80 / 725(11.0)$ & $64 / 448(14.3)$ & $24 / 253(9.5)$ & \\
\hline \multicolumn{5}{|l|}{ Gender } \\
\hline Male & $358 / 725(49.4)$ & $202 / 448(45.1)$ & $104 / 253(41.1)$ & 0.0570 \\
\hline Female & $367 / 725(50.6)$ & $246 / 448(54.9)$ & $149 / 253(58.9)$ & \\
\hline Province (GDP per capita) ${ }^{a}$ & & & & $<0.0001$ \\
\hline High & $234 / 725(44.7)$ & $182 / 448(40.6)$ & $108 / 253(42.7)$ & \\
\hline Low & $491 / 725(67.7)$ & $266 / 448(59.4)$ & 145/253 (57.3) & \\
\hline Residence & & & & $<0.0001$ \\
\hline Urban & $432 / 725(59.6)$ & $196 / 448(43.8)$ & $65 / 253(25.7)$ & \\
\hline Rural & $293 / 725(40.4)$ & $252 / 448(56.2)$ & $188 / 253(74.3)$ & \\
\hline Educational level & & & & $<0.0001$ \\
\hline$\leq$ Primary & $333 / 725(45.9)$ & $257 / 448(53.4)$ & $172 / 253(68.0)$ & \\
\hline Middle/high school & $353 / 725(48.7)$ & $166 / 448(37.1)$ & $74 / 253(29.3)$ & \\
\hline College/higher & $39 / 725(5.4)$ & $25 / 448(5.6)$ & $7 / 253(2.8)$ & \\
\hline Personal monthly income [yuan] & & & & $<0.0001$ \\
\hline$<5,000$ & $221 / 725(30.5)$ & $202 / 448(45.1)$ & $155 / 253(61.3)$ & \\
\hline $5,000-20,000$ & 207/725 (28.6) & $143 / 448(31.9)$ & $60 / 253(23.7)$ & \\
\hline$>20,000$ & $297 / 725(41.0)$ & $103 / 448(23.0)$ & $38 / 253(15.0)$ & \\
\hline \multicolumn{5}{|l|}{ Avenues $^{b}$} \\
\hline$<2$ & $341 / 725(47.0)$ & $238 / 448(53.1)$ & $152 / 253(60.1)$ & 0.0011 \\
\hline $2-4$ & $345 / 725(47.6)$ & 199/448 (44.4) & $94 / 253(37.2)$ & \\
\hline$>5$ & $39 / 725(5.4)$ & $11 / 448(2.5)$ & $7 / 253(2.8)$ & \\
\hline \multicolumn{5}{|l|}{ Previous heart disease } \\
\hline No & $604 / 725(83.3)$ & $377 / 448(84.2)$ & $219 / 253(86.6)$ & 0.4756 \\
\hline Yes & $121 / 725(16.6)$ & $71 / 448(15.9)$ & $34 / 253(13.4)$ & \\
\hline \multicolumn{5}{|l|}{ Previous diabetes mellitus $^{c}$} \\
\hline No & $571 / 724(78.9)$ & $365 / 448(81.5)$ & $208 / 253(82.2)$ & 0.3843 \\
\hline Yes & $153 / 724(21.1)$ & $83 / 448(18.5)$ & $45 / 253(17.8)$ & \\
\hline \multicolumn{5}{|l|}{ Previous hypertension } \\
\hline No & $251 / 725(34.6)$ & $138 / 448(30.8)$ & $69 / 253(27.3)$ & 0.0757 \\
\hline Yes & $474 / 725(65.4)$ & $310 / 448(69.2)$ & $184 / 253(72.7)$ & \\
\hline Previous hyperlipidemia & & & & 0.0544 \\
\hline No & $510 / 725(70.3)$ & $295 / 448(65.9)$ & $188 / 253(74.3)$ & \\
\hline Yes & $215 / 725$ (29.7) & $153 / 448$ (34.2) & 65/253 (25.7) & \\
\hline
\end{tabular}


Table 1 cont. Baseline data of patients in different grades of hospital in China

\begin{tabular}{|c|c|c|c|c|}
\hline Patients & Tertiary & Secondary & Primary hospitals & P-value \\
\hline Family history of stroke & & & & $<0.0001$ \\
\hline No & $534 / 725(73.7)$ & $314 / 448(70.0)$ & $200 / 253(79.1)$ & \\
\hline Yes & $146 / 725(20.1)$ & $128 / 448(28.6)$ & $50 / 253(19.8)$ & \\
\hline Unknown & $45 / 725(6.2)$ & $6 / 448(1.3)$ & $3 / 253(1.2)$ & \\
\hline Stroke in others ${ }^{d}$ & & & & 0.0034 \\
\hline No & $429 / 725(59.2)$ & $302 / 448(67.4)$ & $173 / 253(68.4)$ & \\
\hline Yes & $296 / 725(40.8)$ & $146 / 448(32.6)$ & $80 / 253$ (31.6) & \\
\hline Ways in which patient obtains medical care & & & & $<0.0001$ \\
\hline Hospitalisation & $550 / 725(75.9)$ & $372 / 448(83.0)$ & $152 / 253(60.1)$ & \\
\hline Emergency & $103 / 725(14.2)$ & $15 / 448(3.4)$ & $1 / 253(0.4)$ & \\
\hline Outpatient & $72 / 725(9.9)$ & $61 / 448(13.6)$ & $86 / 253(34.0)$ & \\
\hline GP & $0 / 725(0.0)$ & $0 / 448(0.0)$ & $14 / 253(5.5)$ & \\
\hline$m R S$ score ${ }^{e}$ & & & & $<0.0001$ \\
\hline 0 & $239 / 725(33.0)$ & $136 / 448(30.4)$ & $116 / 253(45.9)$ & \\
\hline 1 & $349 / 725(48.1)$ & $223 / 448(49.8)$ & $127 / 253(50.2)$ & \\
\hline 2 & $89 / 725(12.3)$ & $56 / 448(12.5)$ & $8 / 253(3.2)$ & \\
\hline 3 & $40 / 725(5.5)$ & $25 / 448(5.6)$ & $0 / 253$ & \\
\hline 4 & $6 / 725(0.8)$ & $8 / 448$ (1.8) & $1 / 253(0.4)$ & \\
\hline 5 & $2 / 725(0.2)$ & $0 / 448$ & $1 / 253(0.4)$ & \\
\hline Living status $^{f}$ & & & & 0.1610 \\
\hline With family & $684 / 725(94.3)$ & $410 / 448(91.5)$ & $234 / 253(92.5)$ & \\
\hline With others & $41 / 725(5.7)$ & $38,448(8.5)$ & $19,253(7.5)$ & \\
\hline
\end{tabular}

and CAR was marginal. This indicates that public education concerning secondary prevention of stroke in China still requires improvement $[30,31]$.

According to previous research [24, 32, 33], individuals with higher personal incomes tend to know more about stroke symptoms, and those with higher incomes consistently exhibit greater concerns about their health [17]. Similarly, in our study, patients with higher personal incomes were significantly more likely to have better stroke knowledge. This might be associated with the unequal distribution of healthcare resources in China [34]. As shown in our study, patients with higher incomes are more inclined to be treated in tertiary hospitals. Thus, they might have more stroke knowledge because of the better in-hospital education available in those institutions. Furthermore, high-income patients tend to exhibit higher levels of education and greater awareness of health and thus may have accumulated more health-related knowledge. In contrast, according to our research, patients with lower incomes may not only lack knowledge regarding strokes, but may also favour primary or secondary hospitals.
Therefore, in-hospital education in primary and secondary hospitals needs to be improved and adapted in order to raise awareness among people with lower income levels. To this end, there is a need for education campaigns specifically directed at low-income populations. Furthermore, the construction of 'emergency green channels' to provide timely and efficient care to stroke patients in hospital emergency departments should be prioritised [20, 28].

Surprisingly, residents in economically more developed provinces were less likely to use the EMS immediately following stroke onset than were residents in less economically developed provinces. There may be several explanations for this phenomenon. Firstly, populations in economically advanced regions tend to own cars and have access to advanced transport systems, making it convenient to use medical facilities other than the EMS, such as outpatient services [14, 15, 35]. Secondly, many studies have shown that the main challenges in stroke management in China are pre-hospital delays and a relatively low thrombolysis rate, while the main benefits of the EMS are quicker triage and higher rates of thrombolysis 


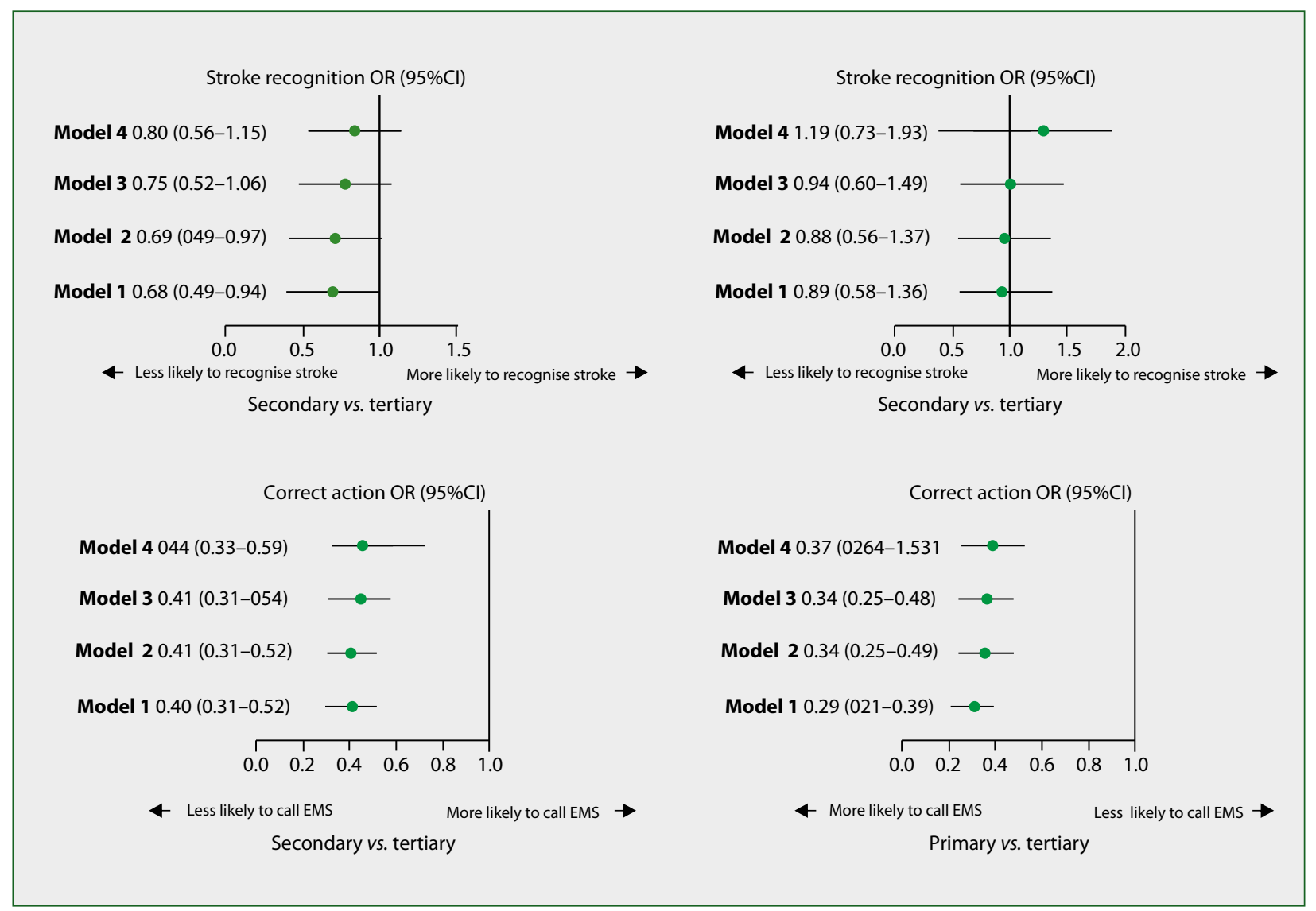

Figure 2. Different logistic regression models presenting associations between hospital classification and stroke recognition or calling EMS. Model 1: Unadjusted; Model 2: Adjusted by age, gender, hospital location, hospital type and cerebral vascular disease, hypertension, diabetes, and dyslipidemia; Model 3: Adjusted by age, gender, hospital location, hospital type, cerebral vascular disease, hypertension, diabetes and dyslipidemia, educational status, income status, living status, and number of ways to learn about stroke; Model 4: Adjusted by age, gender, hospital location, hospital type, cerebral vascular disease, hypertension, diabetes and dyslipidemia, educational status, income status, living status, number of avenues to learn about stroke, stroke in family and community, and approach to seeking medical intervention. $\mathrm{Cl}$ - confidence interval; EMS - Emergency Medical Services; OR - odds ratio

Table 2. Rate of stroke awareness and rate of correct response after acute stroke in patients of primary/secondary/tertiary hospital

\begin{tabular}{|c|c|c|c|c|}
\hline Rate & Tertiary & Secondary & Primary hospitals & P-value \\
\hline Stroke awareness n/N (\%) [95\% OR] & $\begin{array}{c}631 / 725(87.9) \\
{[85.5-90.2]}\end{array}$ & $\begin{array}{c}372 / 448(83.0) \\
{[79.6-86.5]}\end{array}$ & $\begin{array}{c}219 / 253(86.6) \\
{[82.4-90.8]}\end{array}$ & 0.0657 \\
\hline Correct response n/N (\%) [95\% OR] & $\begin{array}{c}577 / 725(79.6) \\
{[76.7-82.5]}\end{array}$ & $\begin{array}{c}272 / 448(60.7) \\
{[56.2-65.2]}\end{array}$ & $\begin{array}{c}134 / 253(53.0) \\
{[46.8-59.3]}\end{array}$ & $<0.0001$ \\
\hline
\end{tabular}

$\mathrm{OR}-$ odds ratio

$[27,36]$. Pre-hospital delays are often substantial in China, even with the availability of emergency departments, because of staff shortages and a lack of training [37]. Also, health education regarding EMS usage following stroke onset is inadequate $[38,39]$. Therefore, some people in economically developed provinces may prefer to use more convenient private transport rather than the EMS to reach hospital after stroke onset.

Another concern is that the patients in wealthier areas may not always have advanced health awareness. It has been suggested that health awareness might, in fact, be greater among individuals in some less developed regions than in high- and middle-income regions. [40]. To explore other possible reasons for this result, further analysis and research are required. The relatively low rate of EMS usage in economically developed regions should be noted by the government and relevant policymakers. Coordinated efforts by the government, community and hospital leaders, and doctors are required to support public education regarding strokes and secondary prevention in some more economically developed provinces. Social media can be a valuable tool to promote health awareness among people in economically developed regions $[7,41]$. 
Table 3. Stroke recognition rate (SRR) and correct action rate (CAR) in individuals with different ways of obtaining medical care among patients hospitalised once

\begin{tabular}{lccccc} 
Rate & Hospitalisation & Emergency & Outpatient & GP & P-value \\
\hline Stroke awareness n/N (\%) & $921 / 1,074$ & $111 / 119$ & $185 / 219$ & $11 / 14$ & $(78.6)$ \\
& $(85.8)$ & $(93.3)$ & $(84.5)$ & 0.0945 & $(15)$ \\
Correct response n/N (\%) & $719 / 1,074$ & $105 / 119$ & $(69.9)$ & $(88.2)$ & $(42.9)$
\end{tabular}

Table 4. Stroke recognition rate (SRR) and correct action rate (CAR) of patients in provinces with high or low gross domestic product (GDP) per capita* and with or without high income level and high educational level among tertiary hospital group

\begin{tabular}{lcc} 
Rate/location & High GDP per capita & Low GDP per capita \\
\hline Stroke awareness n/N (\%) & $206 / 234(88.0)$ & $431 / 491(87.8)$ \\
Correct response n/N (\%) & $174 / 234(74.4)$ & $403 / 491(82.1)$ \\
Rate/income & $<20,000$ yuan/month & $\geq 20,000$ yuan/month \\
Stroke awareness n/N (\%) & $366 / 428(85.5)$ & $271 / 297(91.2)$ \\
Correct response n/N (\%) & $331 / 428(77.3)$ & $246 / 297(82.8)$ \\
Rate/education & $\geq$ College & 0.0201 \\
Stroke awareness n/N (\%) & $36 / 39(92.3)$ & 0.0712 \\
Correct response n/N (\%) & $29 / 39(74.4)$ & $601 / 686(87.6)$
\end{tabular}

*31 provinces in China ranked by their GDP per capita in 2016; those ranking 1-10 are defined as high, those ranking 11-31 are defined as low

It should be recognised that this study has several limitations. Firstly, analysis bias may be present because of the imbalance in the participating hospitals. Approximately 50\% of patients analysed in this study were in tertiary hospitals, which is slightly inconsistent with actual patient distribution as demonstrated in previous research [20,42]. Additionally, our study is based on a questionnaire survey which is designed to evaluate the individuals' intentions, rather than recording real-life responses. Thus, the results are not conclusive regarding the association between hospital classification and patient knowledge about stroke and EMS. Additionally, there is a need for research on in-hospital education regarding stroke in China: hence, this study does not examine the nature of in-hospital education programmes implemented in primary, secondary or tertiary hospitals that are aimed at improving stroke recognition and/or appropriate responses. Besides, there is a lack of data on the quality of in-hospital education at each centre in this research. There has been little research into how in-hospital education regarding strokes is delivered. A fuller understanding of in-hospital education of stroke knowledge could be a future research direction. Finally, the multistage, non-random sampling design and selection in the CNSSS could bias the rates of stroke recognition, EMS usage, and their associated factors [11].

\section{Conclusions}

This study demonstrates that the classification of a hospital is significantly associated with a patient's correct response rate to stroke onset in China. It is vital for medical staff in primary and secondary hospitals to inform their patients about the importance of well-timed EMS utilisation following the onset of stroke symptoms so as to reduce pre-hospital delay. Emergency patients had a significantly higher CAR compared to patients who sought other medical care services in tertiary hospitals.

This suggests insufficient public awareness of EMS usage in China. Therefore, not only must public education on stroke recognition be improved, but prompt EMS usage must also be vigorously advocated in order to reduce pre-hospital delay.

Acknowledgments: We thank all individuals who have contributed to this study and the China Stroke Prevention Commission and the FAST-RIGHT investigators and coordinators. We would like to thank Editage [www.editage.cn] for assistance with English language editing. Finally, we address special thanks to Baohua Chao, Lei Cao, Lingxiao Wang and their team for crucial support in the undertaking of this programme.

Conflict of interest: None.

Funding: This study was funded by the Ministry of Finance of the People's Republic of China [Issued by Finance and Social Security (2016) Document No.50, Ministry of Finance], and Capital Medical Development Research Funding of China (No. 2020-1-4012), and National Key Research and Development Programme of China (No. 2016 YFC0901004).

Ethical permission: The FAST-RIGHT study was approved by the central ethics committee of Peking Union Medical College Hospital (the principal study centre), and all participants provided written informed consent. 


\section{References}

1. Liu L, Liu J, Wang Y, et al. Substantial improvement of stroke care in China. Stroke. 2018; 49(12): 3085-3091, doi: 10.1161/STROKEAHA.118.022618, indexed in Pubmed: 30571434.

2. Pandian JD, Gall SL, Kate MP, et al. Prevention of stroke: a global perspective. Lancet. 2018; 392(10154): 1269-1278, doi: 10.1016/ S0140-6736(18)31269-8, indexed in Pubmed: 30319114.

3. Pan $\mathrm{Y}$, Zhao $\mathrm{X}$, Jiang $\mathrm{Y}$, et al. Prevalence, knowledge, and treatment of transient ischemic attacks in China. Neurology. 2015; 84(23): 2354-2361, doi: 10.1212/WNL.0000000000001665, indexed in Pubmed: 25957333.

4. Yin $\mathrm{X}$, Yang $\mathrm{T}$, Gong $\mathrm{Y}$, et al. Determinants of emergency medical services utilization among acute ischemic stroke patients in Hubei province in China. Stroke. 2016; 47(3): 891-894, doi: 10.1161/STROKEAHA.115.011877, indexed in Pubmed: 26768208.

5. Patel A, Fang J, Gillespie C, et al. Awareness of stroke signs and symptoms and calling 9-1-1 among US adults: national health interview survey, 2009 and 2014. Prev Chronic Dis. 2019; 16: E78, doi: 10.5888/pcd16.180564, indexed in Pubmed: 31228234.

6. Yang J, Zheng M, Chen S, et al. A survey of the perceived risk for stroke among community residents in western urban China. PLoS One. 2013; 8(9): e73578, doi: 10.1371/journal.pone.0073578, indexed in Pubmed: 24039989.

7. Barber SL, Borowitz M, Bekedam H, et al. The hospital of the future in China: China's reform of public hospitals and trends from industrialized countries. Health Policy Plan. 2014; 29(3): 367-378, doi: 10.1093/heapol/czt023, indexed in Pubmed: 23612847.

8. Korda $\mathrm{H}$, Itani Z. Harnessing social media for health promotion and behavior change. Health Promot Pract. 2013; 14(1): 15-23, doi: 10.1177/1524839911405850, indexed in Pubmed: 21558472.

9. Longde $\mathrm{W}$, Ling $\mathrm{Y}$, Yang $\mathrm{H}$, et al. Fixed-dose combination treatment after stroke for secondary prevention in China: a national community-based study. Stroke. 2015; 46(5): 1295-1300, doi: 10.1161/STROKEAHA.114.007384, indexed in Pubmed: 25782466.

10. National Center for Stroke Control and Prevention, t.N.H.C. The China National Stroke screening survey guidelines 2016.

11. Li S, Cui LY, Anderson C, et al. FAST-RIGHT Investigators and Coordinators. Public awareness of stroke and the appropriate responses in China: a cross-sectional community-based study (FAST-RIGHT). Stroke. 2019; 50(2): 455-462, doi: 10.1161/STROKEAHA.118.023317, indexed in Pubmed: 32125134.

12. Harbison J, Hossain O, Jenkinson D, et al. Diagnostic accuracy of stroke referrals from primary care, emergency room physicians, and ambulance staff using the face arm speech test. Stroke. 2003; 34(1): 71-76, doi: $10.1161 / 01 . s t r .0000044170 .46643 .5 e$, indexed in Pubmed: 12511753.

13. Li S, Cui LY, Anderson C, et al. FAST-RIGHT Investigators and Coordinators. Barriers from calling ambulance after recognizing stroke differed in adults younger or older than 75 years old in China. BMC Neurol. 2019; 19(1): 283, doi: 10.1186/s12883-019-1480-6, indexed in Pubmed: 31718577.

14. Li Y, Li C, Xu J, et al. Emergency department enlargement in China: exciting or bothering. J Thorac Dis. 2016; 8(5): 842-847, doi: 10.21037/jtd.2016.03.19, indexed in Pubmed: 27162657.

15. Zhang JF, Qiu MY, Zhang YL, et al. Neurology practice and stroke services across East China: a multi-site, county-level hospital-based survey. BMC Neurol. 2019; 19(1): 293, doi: 10.1186/s12883-019-1518-9, indexed in Pubmed: 31744462.
16. Liu X, Kong D, Lian H, et al. Distribution and predictors of hospital charges for haemorrhagic stroke patients in Beijing, China, March 2012 to February 2015: a retrospective study. BMJ Open. 2018; 8(3): e017693, doi: 10.1136/bmjopen-2017-017693, indexed in Pubmed: 29602836.

17. Fiscella K. Relationships between income, health behaviors, and life expectancy. JAMA. 2016; 316(8): 880, doi: 10.1001/jama.2016.9376, indexed in Pubmed: 27552625.

18. Pickett KE, Wilkinson RG. Income inequality and health: a causal review. Soc Sci Med. 2015; 128: 316-326, doi: 10.1016/j.socscimed.2014.12.031, indexed in Pubmed: 25577953.

19. Johnston DW, Lordan G, Shields MA, et al. Education and health knowledge: evidence from UK compulsory schooling reform. Soc Sci Med. 2015; 127: 92-100, doi: 10.1016/j.socscimed.2014.10.026, indexed in Pubmed: 25459203.

20. Jiang B, Ru X, Sun H, et al. Pre-hospital delay and its associated factors in first-ever stroke registered in communities from three cities in China. Sci Rep. 2016; 6: 29795, doi: 10.1038/srep29795, indexed in Pubmed: 27411494.

21. Schneider AT, Pancioli AM, Khoury JC, et al. Trends in community knowledge of the warning signs and risk factors for stroke. JAMA. 2003; 289(3): 343-346, doi: 10.1001/jama.289.3.343, indexed in Pubmed: 12525235.

22. Teuschl Y, Brainin M. Stroke education: discrepancies among factors influencing prehospital delay and stroke knowledge. Int J Stroke. 2010; 5(3): 187-208, doi: 10.1111/j.1747-4949.2010.00428.x, indexed in Pubmed: 20536616.

23. Nicol MB, Thrift AG. Knowledge of risk factors and warning signs of stroke. Vasc Health Risk Manag. 2005; 1(2): 137-147, doi: 10.2147/ vhrm.1.2.137.64085, indexed in Pubmed: 17315400.

24. Wang MD, Wang Y, Mao L, et al. Acute stroke patients' knowledge of stroke at discharge in China: a cross-sectional study. Trop Med Int Health. 2018; 23(11): 1200-1206, doi: 10.1111/tmi.13148, indexed in Pubmed: 30178470.

25. Yang J, Zheng M, Cheng S, et al. Knowledge of stroke symptoms and treatment among community residents in Western Urban China. J Stroke Cerebrovasc Dis. 2014; 23(5): 1216-1224, doi: 10.1016/j. jstrokecerebrovasdis.2013.10.019, indexed in Pubmed: 24274934.

26. Fussman C, Rafferty AP, Lyon-Callo S, et al. Lack of association between stroke symptom knowledge and intent to call 911: a population-based survey. Stroke. 2010; 41(7): 1501-1507, doi: 10.1161/STROKEAHA.110.578195, indexed in Pubmed: 20466995.

27. Zhou Y, Yan S, Song X, et al. Intravenous thrombolytic therapy for acute ischemic stroke in Hubei, China: a survey of thrombolysis rate and barriers. BMC Neurol. 2019; 19(1): 202, doi: 10.1186/s12883-0191418-z, indexed in Pubmed: 31438899.

28. Fassbender K, Balucani C, Walter S, et al. Streamlining of prehospital stroke management: the golden hour. Lancet Neurol. 2013; 12(6): 585-596, doi: 10.1016/S1474-4422(13)70100-5, indexed in Pubmed: 23684084.

29. Rankin P, Marsh D, McLaughlin J. Abstract WP373: Emergency medical service stroke education in a rural community: need for improvement. Stroke. 2017; 48(Suppl 1), doi: 10.1161/str.48.suppl_1.wp373.

30. Liu L, Wang D, Wong KS, et al. Stroke and stroke care in China: huge burden, significant workload, and a national priority. Stroke. 2011; 42(12): 3651-3654, doi: 10.1161/STROKEAHA.111.635755, indexed in Pubmed: 22052510.

31. Wang YJ, Zhang SM, Zhang L, et al. Chinese guidelines for the secondary prevention of ischemic stroke and transient ische- 
mic attack 2010. CNS Neurosci Ther. 2012; 18(2): 93-101, doi: 10.1111/j.1755-5949.2011.00290.x, indexed in Pubmed: 22313945.

32. Kim YS, Park SS, Bae HJ, et al. Public awareness of stroke in Korea: a population-based national survey. Stroke. 2012; 43(4): 1146-1149, doi: 10.1161/STROKEAHA.111.638460, indexed in Pubmed: 22156687.

33. Bay JL, Spiroski AM, Fogg-Rogers L, et al. Stroke awareness and knowledge in an urban New Zealand population. J Stroke Cerebrovasc Dis. 2015; 24(6): 1153-1162, doi: 10.1016/j.jstrokecerebrovasdis.2015.01.003, indexed in Pubmed: 25847304.

34. Li D, Zhou Z, Si Y, et al. Unequal distribution of health human resource in mainland China: what are the determinants from a comprehensive perspective? Int J Equity Health. 2018; 17(1): 29, doi: 10.1186/ s12939-018-0742-z, indexed in Pubmed: 29486791.

35. Wu Na, Zhao S, Zhang Qi. A study on the determinants of private car ownership in China: findings from the panel data. Transportation Research Part A: Policy and Practice. 2016; 85: 186-195, doi: 10.1016/j.tra.2016.01.012.

36. Wu S, Wu Bo, Liu M, et al. China Stroke Study Collaboration. Stroke in China: advances and challenges in epidemiology, prevention, and management. Lancet Neurol. 2019; 18(4): 394-405, doi: 10.1016/ S1474-4422(18)30500-3, indexed in Pubmed: 30878104.
37. Jiang B, Liang S, Peng ZR, et al. Transport and public health in China: the road to a healthy future. Lancet. 2017; 390(10104): 1781-1791, doi: 10.1016/S0140-6736(17)31958-X, indexed in Pubmed: 29047445.

38. Jing Z, Renyu L. Calling for a rapid recognition and response program for stroke in China. Transl Perioper Pain Med. 2016; 1(4): 1-4, doi: 10.31480/2330-4871/042, indexed in Pubmed: 28105445.

39. Sun $\mathrm{H}$, Chen $\mathrm{S}$, Jiang $\mathrm{B}$, et al. Public knowledge of stroke in Chinese urban residents: a community questionnaire study. Neurol Res. 2011; 33(5): 536-540, doi: 10.1179/016164111X13007856084368, indexed in Pubmed: 21669124.

40. Palafox B, McKee M, Balabanova D, et al. Wealth and cardiovascular health: a cross-sectional study of wealth-related inequalities in the awareness, treatment and control of hypertension in high-, middleand low-income countries. Int J Equity Health. 2016; 15(1): 199, doi: 10.1186/s12939-016-0478-6, indexed in Pubmed: 27931255.

41. Lyson HC, Le GM, Zhang J, et al. Social media as a tool to promote health awareness: results from an online cervical cancer prevention study. J Cancer Educ. 2019; 34(4): 819-822, doi: 10.1007/s13187018-1379-8, indexed in Pubmed: 29948924.

42. Zhou Y, Yang $T$, Gong $Y$, et al. Pre-hospital delay after acute ischemic stroke in central urban China: prevalence and risk factors. Mol Neurobiol. 2017; 54(4): 3007-3016, doi: 10.1007/s12035-016-9750-4, indexed in Pubmed: 27032390. 